\title{
Interlaboratory collaboration to determine the performance of the Randox food diagnostics biochip array technology for the simultaneous quantitative detection of seven mycotoxins in feed
}

\author{
L. Sibanda ${ }^{*}$, K. McCallum ${ }^{2}$, M. Plotan ${ }^{1}$, S. Webb ${ }^{3}$, B. Snodgras ${ }^{4}$, Q. Muenks ${ }^{5}$, J. Porter ${ }^{1}$ and P. Fitzgerald ${ }^{1}$ \\ ${ }^{1}$ Randox Food Diagnostics Ltd., 55 Diamond Road, Crumlin, BT29 4QY, United Kingdom; ${ }^{2}$ Colorado Department of \\ Agriculture, Division of Laboratory Services - Biochemistry Laboratory, 300 South Technology Court, Broomfield, CO \\ 80021, USA; ${ }^{3}$ University of Kentucky, Division of Regulatory Services, 103 Regulatory Services Bldg, Lexington, KY 40546, \\ USA ${ }^{4}$ American Association of Feed Control Officials (AAFCO), Proficiency Testing Program, 1800 S. Oak Street, Suite \\ 100, Champaign, IL 61820, USA; ${ }^{5}$ Missouri Department of Agriculture, Bureau of Feed and Seed, 115 Constitution Drive, \\ Jefferson City, MI 65109, USA; liberty.sibanda@randox.com
}

Received: 19 March 2021 / Accepted: 9 August 2021

() 2021 Wageningen Academic Publishers

\section{OPEN ACCESS @ (i) RESEARCH ARTICLE}

\begin{abstract}
An inter-laboratory collaborative study was performed to evaluate the performance of the Biochip Array Technology (BAT) Myco 7 method. The Myco 7 Array is a method which simultaneously and quantitatively detects 20 mycotoxins including aflatoxins $B_{1}, B_{2}, G_{1}$ and $G_{2}$, ochratoxin $A$, deoxynivalenol, zearalenone, fumonisin $B_{1}, B_{2}$ and $B_{3}$ and $T-2$ and HT-2 toxin. The BAT Myco 7 method was collaboratively evaluated by nine government and private Association of American Feed Control Officials (AAFCO) laboratories. Samples were analysed in a proficiency testing round format. Seventeen blind samples were analysed on the same equipment using Myco 7 kits. $99 \%$ of the results fell within an acceptable $Z$-score range of $-2|<Z<|+2$. Deoxynivalenol had a $100 \% Z$-score pass rate, while a $99 \%$ pass was recorded for aflatoxins, zearalenone, ochratoxin A and fumonisins. T-2 toxin had a 97\% Z-score pass rate. HorRat analysis for reproducibility used a range of $0.3<\mid$ HorRat $\mid \leq 2$. The target was met for deoxynivalenol, zearalenone, T-2 and HT- 2 toxin, and aflatoxins $B_{1}, B_{2}, G_{1}$ and $G_{2}$ assays. Fumonisins and ochratoxin A assays had a $93 \%$ and $94 \%$ pass, respectively. The reproducibility co-efficiency of variation was between 16 and $20 \%$ meeting set criterion of $<40 \%$ and is, therefore, fit-for-purpose for use in the AAFCO control programs for mycotoxins.
\end{abstract}

Keywords: biochip, mycotoxins, multiplexed, inter-laboratory, HorRat, Z-scores

\section{Introduction}

Mycotoxins are fungal secondary metabolites produced in different types of food matrices. There are a number of toxigenic moulds, however, the most important ones are Aspergillus flavus, Aspergillus niger, Aspergillus ochraceous, Aspergillus parasiticus, and Aspergillus verrucosum which are responsible for the production of aflatoxins $B_{1}, B_{2}, G_{1}$ and $G_{2},\left(\mathrm{AFB}_{1}, \mathrm{AFB}_{2} \mathrm{AFG}_{1}\right.$, and $\mathrm{AFG}_{2}$, respectively) as well as ochratoxin A (OTA) among them. The other important genus regarding toxigenicity is Fusarium, notably Fusarium graminearum, Fusarium culmorum, Fusarium verticilloides, and Fusarium proliferatum which among themselves are responsible for the production of deoxynivalenol (DON), zearalenone (ZEN), fumonisins $\mathrm{B}_{1}, \mathrm{~B}_{2}, \mathrm{~B}_{3}\left(\mathrm{FB}_{1}\right.$, $\mathrm{FB}_{2}, \mathrm{FB}_{3}$, respectively) and T-2 and HT-2 toxin (T-2 and HT-2, respectively). The incidence of toxigenic fungi and subsequent production of mycotoxins are now a worldwide occurrence and are observed in food and feed due to suitable weather and climate, inappropriate production and storage practices for crops. Aflatoxins, fumonisins, ochratoxins, trichothecenes, and ZEN are mycotoxins of agroeconomic importance. In Europe, contamination with mycotoxins can occur in as high as $75-100 \%$ of animal feed samples (Streit et al., 2012). Following a three year worldwide study it was found that $81 \%$ of 23,781 samples were positive for at least 
one mycotoxin (Rodrigues and Naehrer, 2012). Worldwide mycotoxin contamination situation is very similar. Annual Biomin reports have shown that more than $90 \%$ of various tested samples contained different types of mycotoxins (Biomin, 2019). The most recent survey, summarising year 2019 included over 20,000 samples and reported 91 and $71 \%$ being contaminated with at least 1 and more than 1 mycotoxin, respectively (Biomin, 2019).

The occurrence of mycotoxins in food and feed is now high on the agenda regarding to human and animal exposure. Therefore, countries around the world implement mycotoxin regulations which, in most cases, are based on the Codex Alimentarius of the Food and Agricultural Organization (FAO). However, some countries have set their own maximum tolerance limits based on toxicity data which determines the daily exposure rates, based on their local diets. This in turn determines the maximum tolerance limits. When comparing the various mycotoxin regulations worldwide, the European Union seems to have the lowest maximum residue limits (MRLs) for mycotoxins. Other well-known jurisdictions with their own mycotoxin tolerance limits include the United States of America. In order to limit the secondary exposure of humans to mycotoxins through agricultural products, feed and feed products must be tested for mycotoxin contamination. In the USA the sale and distribution of animal feed is regulated by members of the American Association of Feed Control Officials (AAFCO).

Sensitive and accurate methods of analysis are required for the effective management and control of mycotoxins. As a general principle, mycotoxins should be isolated, and separated from the matrix through sample extraction procedures which traditionally involve organic solvents, and now recently some rapid on-site methods using water. Extraction methods include liquid-liquid extraction (LLE), solid-liquid extraction (SLE), Quick Easy Cheap Rough and Safe (QuEChERS), solid phase extraction (SPE), immunoaffinity columns (IACs), molecularly imprinted polymers (MIPs) and aptamer-affinity columns (AACs) (Alshannaq and $\mathrm{Yu}, 2017)$. These extraction methods are used in various mycotoxin testing methods, and range from simple on-site rapid test, laboratory-based enzymelinked immunoassays (ELISA), high performance liquid chromatography (HPLC) as well as liquid chromatography mass/spectrometry (LC-MS/MS).

Immunoassay-based methods, like lateral-flow dipsticks (LFDs) and ELISA are useful when rapid analysis of mycotoxins is critical to provide the mycotoxin status of a sample (Krska and Molinelli, 2008). Traditionally LFDs, ELISAs and HPLC are single mycotoxin detection techniques and hence a need for the development of simple rapid multi-mycotoxin detection techniques. HPLC and LC-MS/MS methods are currently the main techniques used for the quantitative detection of mycotoxins. Most
HPLC methods used routinely are only capable of detecting multiple mycotoxins within a family or group, for example, the aflatoxin group $\left(\mathrm{AFB}_{1}, \mathrm{AFB}_{2}, \mathrm{AFG}_{1}\right.$, and $\left.\mathrm{AFG}_{2}\right)$. This is due to the fact that each type of mycotoxin has a specific optimum light absorption within either UV wavelengths or fluorescence wavelength of HPLC detectors. This has, therefore, influenced the development and routine use of single mycotoxin HPLC methods. However, Curticapean et al. (2011) described the development of a multi-mycotoxin method detecting $\mathrm{AFB}_{1}$, OTA and ZEN in maize. They used a Dionex Ultimate 3000 system with a UV Detector capable of simultaneous detection on four different channels. The limit of quantification achieved for $\mathrm{AFB}_{1}$ was $3 \mu \mathrm{g} / \mathrm{kg}$ which fails to cover the $2 \mu \mathrm{g} / \mathrm{kg}$ specified by EU Regulations for most foods. However, Irakli et al. (2017) described the development of a multi-mycotoxin HPLC method for the detection of aflatoxins, DON, OTA and ZEN. They used a multi-mycotoxin immunoaffinity column clean-up procedure followed by chromatographic separation and detection on a Diode-Array and Fluorescence Detectors utilising a postcolumn derivatization step. The game changer though has been the development of multi-mycotoxin detection methods on the LC-MS/MS for both qualitative and quantitative applications (Tittlemier et al., 2019). However, Bazin et al. (2010) reported on a rapid membrane-based test for the detection of OTA and $\mathrm{AFB}_{1}$, which shows that there are efforts to upgrade the capability of these formally single analyte detection techniques to multiple analyte detection.

Animal feed consists of various ingredients with each one contributing at least one mycotoxin on average. Therefore, animal feed products generally contain at least one mycotoxin making it imperative for methods to detect and quantify more than one mycotoxin. In order to control mycotoxins in animal feed a multi-mycotoxin testing strategy is highly recommended. Therefore, AAFCO set out to evaluate the performance of the Biochip Array Technology for the detection of seven groups of mycotoxins $\left(\mathrm{AFB}_{1}, \mathrm{AFB}_{2}\right.$, $\mathrm{AFG}_{1}, \mathrm{AFG}_{2}$, OTA, ZEN, DON, fumonisins (FBs), and T-2/HT-2) using the Myco 7 method. This technology is based on the biochip $(9 \times 9 \mathrm{~mm})$, which is the solid phase and the vessel in which miniaturised chemiluminescence immunoassays take place simultaneously (Fitzgerald et al., 2005; Porter et al., 2012). This technology has been applied elsewhere for the detection of drug residues in food-related products and has been reported by Gaudin et al. (2014) and Plotan et al. (2016). It was Plotan et al. (2016) who described the validation of the Biochip Array Technology for multiplex mycotoxin detection. They developed, and then validated the BAT platform for mycotoxin testing according to the European Commission Decision No. 2002/657/EC (EC, 2002) where they demonstrated low coefficients of variation (CVs) (10.6 and 11.6\%, respectively) for the $r$ and withinlaboratory $\mathrm{R}$ values. They also successfully demonstrated the performance of the test in analysing feed certified reference materials (CRM) samples obtained from the 
Food Analysis Performance Assessment Scheme (FAPAS) program. In their study they positively correlated BAT mycotoxin results for feed with those of LC-MS/MS. The application of BAT technology as functional multi-assays with intended purpose as a semi-quantitative detection method for mycotoxins in feeds was described previously (Berthiller et al., 2018).

Since the Plotan et al. (2016) study was a single laboratory validation, we set out to investigate the reproducibility and benefits of multiplex BAT system as a multi-mycotoxin detection tool across multiple laboratories. Therefore, the main aim of this study was to investigate the performance of the Myco 7 Array against multi-mycotoxin method performance criteria defined by AAFCO in their method needs statement. An interlaboratory collaborative method performance study is carried out for the purposes of new method validation and as a requirement for full validation and standardisation in terms of percent relative standard deviation (\%RSD) which in turn is compared to theoretical Horwitz and HorRat values (De Girolamo et al., 2017; Taverniers et al., 2004). The interlaboratory collaborative study generated results indicating whether or not the method under investigation is fit-for-purpose under the set criteria for proficiency testing and reproducibility in different laboratory conditions.

\section{Materials and methods}

\section{Kit reagents and solvents}

The Myco 7 Biochip Array kit Cat. No.: EV4065 (for simultaneous detection of DON, HT/T-2, ZEN, OTA, $\mathrm{AFB}_{1}, \mathrm{AFB}_{2}, \mathrm{AFG}_{1}, \mathrm{AFB}_{2}, \mathrm{FB}_{1}, \mathrm{FB}_{2}$, and $\mathrm{FB}_{3}$ ) was obtained from Randox Food Diagnostics Ltd., Crumlin, UK. The kit contains multianalyte biochips, assay diluent, conjugate diluent, multianalyte conjugate, multianalyte calibrators (spanning the range of each assay), multianalyte control, signal reagent, washing buffer, calibration disc, and barcodes. The multianalyte calibrators included all the analytes used for the standardisation of each of seven competitive immunoassays on the biochip at nine different concentration levels, with the following typical calibration ranges for sensitive detection and monitory level, respectively: 0-4.5 and 0-56.25 $\mu \mathrm{g} / \mathrm{kg}$ expressed as $\mathrm{AFB}_{1}$ equivalence for the $\mathrm{AFB}_{1}$ assay, 0-30 and 0-375 $\mu \mathrm{g} / \mathrm{kg}$ expressed as $\mathrm{AFG}_{1}$ equivalence for the $\mathrm{AFG}_{1}$ assay, 0-2,000 and $0-25,000 \mu \mathrm{g} / \mathrm{kg}$ expressed as DON equivalence for the DON assay, 0-5,000 and 0-62,500 $\mu \mathrm{g} / \mathrm{kg}$ expressed as $\mathrm{FB}_{1}$ equivalence for the fumonisins assay, $0-20$ and $0-250 \mu \mathrm{g} / \mathrm{kg}$ expressed as OTA equivalence for the OTA assay, 0-80 and $0-1000 \mu \mathrm{g} / \mathrm{kg}$ expressed as T-2 equivalence for T-2 assay, and $0-40$ and $0-500 \mu \mathrm{g} / \mathrm{kg}$ expressed as ZEN equivalence for the ZEN assay. Higher measuring ranges were applied by testing samples at higher dilution. The biochips were supplied in carriers $(3 \times 3$ biochips per carrier), and a carrier handling tray was provided with the system that allows the simultaneous handling of between three samples up to 45 samples (at the end users discretion). Solvents including acetonitrile and methanol (HPLC grade) used for extraction of samples were supplied individually by each laboratory.

\section{Analyser instrument}

Data was generated and processed using the semiautomated benchtop biochip analyser Evidence Investigator (Cat. No.: EV3602; Randox Food Diagnostics). The Evidence Investigator consists of the main imaging console [charge coupled device camera (for chemiluminescence detection)], computer, Investigator imaging software; barcode scanner, 6-carrier holder, and a thermo-shaker.

\section{Feed samples assigned value}

The feed sample CRMs used in this study were obtained from AAFCO's previous proficiency test (PT) rounds. They were purchased by AAFCO from Trilogy Analytical Laboratories (Washington, MO, USA), a reference material producer, which manufactures under the ISO 17034:2016 scope of accreditation. The feed matrices were produced under the sub-category of reference material as organic reference materials, under the International Laboratory Accreditation Cooperation (ILAC) Reference Material Category A3.3 Foodstuffs, and Class; Mycotoxins in Foodstuffs. The method used for mass fraction determination by the reference material producer was LC-MS/MS. The assigned values are detailed in Table 1. The feed matrices used included dairy feed, rabbit feed, dried distillers grains with solubles (DDGS), equine feed, dog food, poultry feed and swine feed. Briefly, feed matrices were ground to a fine consistency of $30 \mathrm{mesh} ; 0.595 \mathrm{~mm}$ and thoroughly homogenised to ensure uniform distribution of the analytes. Samples were mixed by the Paul Schatz method to ensure homogeneity. In order to determine the assigned value, samples were analysed numerous times over the course of several analytical runs utilising the reference method listed above to generate specific concentration data which best estimates the true value as obtained in one laboratory utilising one method.

The samples were then weighed into $50 \mathrm{ml}$ conical centrifuge tubes and shipped to the 9 participating laboratories. The fifteen samples were specifically prepared for the AAFCO Proficiency Testing (PT) Program and their use was extended into this Interlaboratory Collaborative study. Randox Food Diagnostics Ltd provided two additional samples (a Myco 7 kit control and a FAPAS PT sample 04335). Each of the 17 samples were divided into 9 equal fractions and sent to the 9 AAFCO participating laboratories. The instrument was installed at each laboratory and after one day of training an analyst from each laboratory prepared and tested all the samples provided. 
Table 1. Assigned values $(\mu \mathrm{g} / \mathrm{kg})$ for each mycotoxin per certified reference material sample. ${ }^{1}$

\begin{tabular}{lccccccc} 
Sample ID & AFB $_{1}$ & AFG $_{1}$ & OTA & FBs & DON & T-2 & ZEN \\
& & & & & & & \\
MO1 & 37 & 4.241 & 147 & 2,512 & 1,202 & 87.6 & 283 \\
MO2 & 39 & 3.879 & 17 & 1,248 & 2,805 & 266.3 & 565 \\
MO3 & 15 & 2.47 & 221 & 2,900 & 1,886 & 1,223 & 360 \\
MO4 & 15 & 2.17 & 194 & 1,584 & 1,670 & 577.2 & 198 \\
MO5 & 27 & nd & 210 & 6,700 & 1,363 & 105.1 & 174 \\
MO6 & 2.8 & nd & 173 & 9,114 & 1,388 & 93.3 & 173 \\
MO7 & 13 & nd & 330 & 1,897 & 1,431 & 165.1 & 590 \\
MO8 & 10 & nd & 270 & 2,784 & 1,652 & 497.8 & 290 \\
NY1 & 15.1 & nd & 220.3 & 2,923 & 1,886 & $1,227.5$ & 358.3 \\
NY2 & 38.6 & 4.04 & 16.7 & 1,084 & 2,805 & 266.2 & 564.1 \\
NY3 & 150.9 & 10.60 & 15.7 & 6,790 & 11,776 & 346.7 & 1,546 \\
NY4 & 10.1 & nd & 269.6 & 2,644 & 1,652 & 490.5 & 280.1 \\
NY5 & 12.5 & 1.45 & 328.8 & 2,101 & 1,431 & 164.1 & 584.3 \\
NY6 & 27.1 & 2.58 & 208 & 6,097 & 1,363 & 104.4 & 173.3 \\
NY7 & 2.9 & nd & 172.3 & 9,613 & 1,388 & 94.2 & 173 \\
\hline
\end{tabular}

${ }^{1} \mathrm{AFB}_{1}=$ aflatoxin $\mathrm{B}_{1} ; \mathrm{AFG}_{1}=$ aflatoxin $\mathrm{G}_{1} ; \mathrm{DON}=$ deoxynivalenol; $F B s=$ fumonisins $B_{1}, B_{2}$ and $B_{3} ;$ OTA = ochratoxin $A ; T-2=T-2$ toxin; $Z E N=$ zearalenone; $\mathrm{nd}=$ not detected.

\section{Inter-laboratory collaborative validation}

Inter-laboratory collaborative validation is designed to identify factors affecting measurement results among laboratories, to check method transferability to other laboratories, whether the written protocol is clear to new users, and to estimate the precision characteristics of the method in practice. In order to carry-out an interlaboratory collaborative validation a minimum of 8 laboratories is required and that could be reduced to 5 in exceptional circumstances. A minimum of 5 test materials are required, however, this can be reduced to 3 under certain conditions. Further characterisation of method performance under inter-laboratory collaborative conditions have been described previously (ISO 5725-1994; Horwitz, 1994; and the AAFCO Mycotoxin PT Scheme (AAFCO, 2018)).

In an inter-laboratory experiment, different laboratories determine some characteristic, e.g. the concentration of an analyte in one or various homogeneous samples under documented conditions. This collaborative study was used to test the precision performance of a single analytical method (Biochip Array Technology - Myco 7 Test). The principal aim of this study was to evaluate the laboratory performance of the BAT Myco 7 Array against the fitness for purpose criteria set by AOAC/AAFCO. The study was also designed to ascertain the method robustness against possible method or laboratory bias in order to qualify the test for routine use. Participating laboratories included: (1) North Carolina Department of Agriculture \& Consumer Services, Food \& Drug Protection Division, (2) New York State Opportunity, Agriculture \& Markets, (3) Agri-King, (4) Regulatory Services Department, College of Agriculture, Food \& Environment, University of Kentucky, (5) State of Missouri, Department of Agriculture, Feed \& Treated Timber Laboratory, (6) Nebraska Department of Agriculture Laboratory, (7) Minnesota Department of Agriculture, (8) Microbiology Laboratory, Office of Indiana State Chemist, Purdue University and, (9) State Chemical Lab, Feed, Fertilizer \& Lime, Alabama Department of Agriculture \& Industries.

A PT round and Interlaboratory Collaborative study both require a minimum of 8 laboratories to participate. Further, a minimum of $\geq 5$ sample matrices and one specific method are required for a interlaboratory collaborative study. This collaborative study was designed to investigate the fitness-for-purpose of the Biochip Myco 7 Array for the simultaneous detection of 7 mycotoxins in feed matrices across 9 AAFCO laboratories. The format of the study resembled (1) a PT round by providing a specific set of feed matrices with a predetermined concentration (assigned values) for Z-score determination, and (2) an interlaboratory study to evaluate the reproducibility across the 9 laboratories.

The PT characteristics we adopted were (a) provision of sample matrix with assigned values, (b) results were analysed to determine the Z-score for each laboratory and each specific/mycotoxin combination (c) and results were communicated at the internal AAFCO annual conference. The Z-score criterion used was $|-2| \leq|+2|$.

Specific method performance requirement for reproducibility was determined by means of the HorRat value to score the precision of the method across the 9 laboratories for each mycotoxin, the interpretation of which was as follows:

\section{$0.3 \mid<$ HorRat $\leq \mid 2 \quad$ acceptable result}

$0.3 \mid<$ HorRat $\leq \mid 3 \quad$ questionable result

\section{$0.3 \mid<$ HorRat $>\mid 3 \quad$ unacceptable result}

The performance requirements for the method focused on reproducibility, and accuracy as prescribed by the AOAC Method Needs Statement adopted by AAFCO (AOAC, 2009). The method performance criteria stated that the accuracy range needs to be measured by $Z$-score and samples must fall within Z-score range of $-2|<Z<|+2$. Further, the method should have the following operational ranges (Table 2), as prescribed by the AOAC Method Needs Statement, for aflatoxins, fumonisins, DON, T-2/HT-2, OTA and ZEN (AOAC, 2009). 
Table 2. Illustration of the method performance specifications including operational ranges and accuracy requirements for the 7 mycotoxins. $^{1}$

\begin{tabular}{|c|c|c|c|c|c|c|c|}
\hline & DON & $\mathrm{T}-2$ & OTA & ZEN & $\mathrm{AFB}_{1}$ & AF total & FBs \\
\hline Target quantitation level $(\mu \mathrm{g} / \mathrm{kg})$ & 1000 & 100 & 100 & 500 & 5 & 10 & 1000 \\
\hline Operational range $(\mu \mathrm{g} / \mathrm{kg})$ & $100-10,000$ & $10-1000$ & $10-1000$ & $50-5,000$ & $0.5-50$ & $1-100$ & $100-10,000$ \\
\hline Accuracy & $80-110 \%$ & $70-110 \%$ & $70-110 \%$ & $80-110 \%$ & $60-120 \%$ & $60-120 \%$ & $80-110 \%$ \\
\hline
\end{tabular}

All participants of the inter-laboratory study had withinlaboratory quality assurance and quality systems. The method used in this collaborative study was designed to cover a wide range of dilution factors in order to ensure method applicability for the various mycotoxin concentrations encountered in the feed.

\section{Sample preparation}

The generic extraction of mycotoxins was performed following the manufacturer's instructions by mixing $5 \mathrm{~g}$ ground homogenised feed sample with $25 \mathrm{ml}$ of a solvent mixture (acetonitrile/methanol/water; 50:40:10; v/v/v) in a $50 \mathrm{ml}$ centrifuge tube. The sample was mixed on a vortex mixer for $60 \mathrm{~s}$, followed by $10 \mathrm{~min}$ rolling on a tub roller mixer or rotary shaker, and centrifuged for $2 \mathrm{~min}$ at 1,600 relative centrifugal force ( $\mathrm{rcf}$ ). After centrifugation, the supernatant $(50 \mu \mathrm{l})$ was diluted in $150 \mu \mathrm{l}$ of workingstrength wash buffer to analyse the sample at required detection level. Then the diluted sample was applied to the biochip.

\section{Biochip array immunoassay procedure}

The experimental procedure was performed following the manufacturer's instructions. Briefly, $150 \mu \mathrm{l}$ assay diluent was applied to the biochip, followed by $50 \mu \mathrm{l}$ calibrator or sample. After a $30 \mathrm{~min}$ incubation at $25^{\circ} \mathrm{C}$ and 370 rpm in the thermo-shaker, $100 \mu \mathrm{l}$ conjugate was added to each biochip and further incubated for $60 \mathrm{~min}$ at $25^{\circ} \mathrm{C}$ and $370 \mathrm{rpm}$ in the thermo-shaker. The biochips were then washed manually with working strength wash buffer, after which the signal reagent $(250 \mu \mathrm{l})$ was added and incubated for $2 \mathrm{~min}$. The chemiluminescent signal output generated from the seven immunoassays on the biochip was captured using the Evidence Investigator's digital imaging technology. The system uses a dedicated software, which automatically processes, reports, and archives the data generated.

\section{Results}

The collaborative study set-up criteria were met. There were 17 materials analysed by 9 laboratories. All 17 samples were reporting concentrations within required measuring ranges for five assays including OTA, DON, T2, ZEN and AFB . $^{\text {. }}$ There were 15 of 17 samples within measurement ranges for fumonisins and 10 of 17 for $\mathrm{AFG}_{1}$ assay. All samples below measuring ranges were reported as negative, below Myco 7 limit of detection (LOD) and below bottom cut off value set in study requirements. The main aim of the study was to assess accuracy and reproducibility of the method as expressed by the statistical data.

\section{Calibration}

To ensure both accuracy and reproducibility are achieved, the Evidence Investigator Biochip Array Technology simultaneously generated multiple calibration curves each for every analyte being detected as illustrated in Supplementary Figure S1-S7. The automatically set minimum acceptable correlation coefficient for each calibration curve was $95 \%$. Concentrations of each of the mycotoxins under investigation were automatically calculated using these calibration curves.

\section{Accuracy and reproducibility data analysis}

Reproducibility and accuracy was assessed by means of Z-scores since the collaborative study was structured in a PT format and HorRat values. For the purposes of this study a $\mathrm{Z}$-score interval of $-2|<\mathrm{Z}<|+2$ and a HorRat value range of $0.3 \mid<$ HorRat $\leq \mid 2$ and coefficient of variation of $<40 \%$ were used. Reproducibility of the method was expressed and illustrated by the agreement of the mycotoxin concentrations across the laboratories. Z-scores were calculated from a normally distributed 0 centred Z-score using the $\sigma_{f f p}$ based on \%RSD. First $\sigma_{f f p}$ was calculated with the formula:

$\sigma_{f f p}=\frac{X_{\alpha} \times \% R D}{100}$ 
Where $\sigma_{f f p}$ is the target standard deviation; $X_{a}$ is the population average; and $\% R S D$ is relative standard deviation. After determining the $\sigma_{f f p}$ the Z-score was calculated thus:

$$
\mathrm{Z}=\frac{\mathrm{X}_{\mathrm{LAB}}-\mathrm{X}_{\alpha}}{\sigma_{f f p}}
$$

Where $X_{L A B}$ is the result submitted by the laboratory; $X_{a}$ is the population average; and $\sigma_{f f p}$ target standard deviation.

The adoption of an analytical method can be officially approved on the basis of the results of a method's performance in inter-laboratory collaborative studies, and a comparison of these results is an external way of assuring quality control among laboratories concurrently. Figure 1 illustrates how error estimation increases with decreasing concentrations. This would typically affect the HorRat pass rate for mycotoxins, e.g. $\mathrm{AFB}_{1}$ and $\mathrm{AFG}_{1}$ which occur at significantly low concentrations.

\section{Data presentation}

Figures 2 and 3 present samples Z-score results for each laboratory and per each mycotoxin across all 17 tested samples. Within the study there were 966 data points generated and $99 \%$ of these data points were within set $\mathrm{Z}$-score range. There was a $100 \% \mathrm{Z}$-score pass rate among all 9 laboratories for the DON assay. A 99\% passing rate was determined for fumonisins, OTA, ZEN and both aflatoxin assays and a $97 \%$ passing rate for the T-2 assay. In regard to laboratories, there were 3 labs generating all the data and 3 labs having 99\% of their results within Z-score range.
The remaining 2 and 1 laboratories reported 98 and 97\% results within Z-score range, respectively.

Sixteen samples tested across 9 laboratories showed $98 \%$ of data points, a total of 103, being in the set HorRat range of 0.3-2 (Figure 4). There was a $100 \%$ pass for HorRat values for DON, ZEN, T-2/HT-2, $\mathrm{AFB}_{1}$ and $\mathrm{AFG}_{1}$ assays, which illustrated a high reproducibility capability of the method. There were only 2 samples, one for the fumonisins assay and one for the OTA assay which showed HorRat value just out of range being 2.13 and 2.11, respectively. In both instances there was one laboratory, which generated clearly outstanding reading that caused HorRat value increase. A number of samples had $\mathrm{AFG}_{1}$ concentrations $<\mathrm{LOD}$ as well as two samples for the fumonisin assay, therefore, HorRat data could not be computed for them. It was also observed that samples contaminated at lower levels with mycotoxins including majority of samples containing $\mathrm{AFB}_{1}$ and $\mathrm{AFG}_{1}$ showed lower HorRat values, in a range of 0.40.8 . Again, the lower HorRat values observed are most likely caused by possible errors at lower concentrations as illustrated in Figure 1 and not by assay reproducibility. It could also be confirmed by \% $\mathrm{CV}$, which for $\mathrm{AFB}_{1}$ and $\mathrm{AFG}_{1}$ assays were 19.8 and $17.6 \%$, respectively.

HorRat value for kit control sample was not calculated and is not presented due to its very low concentrations across all analytes and limitations that the HorRat equation has at these levels (Horwitz, 1994). The HorRat equation has an exaggerated error rate at low concentrations which results in higher failure rates for samples with low concentrations.

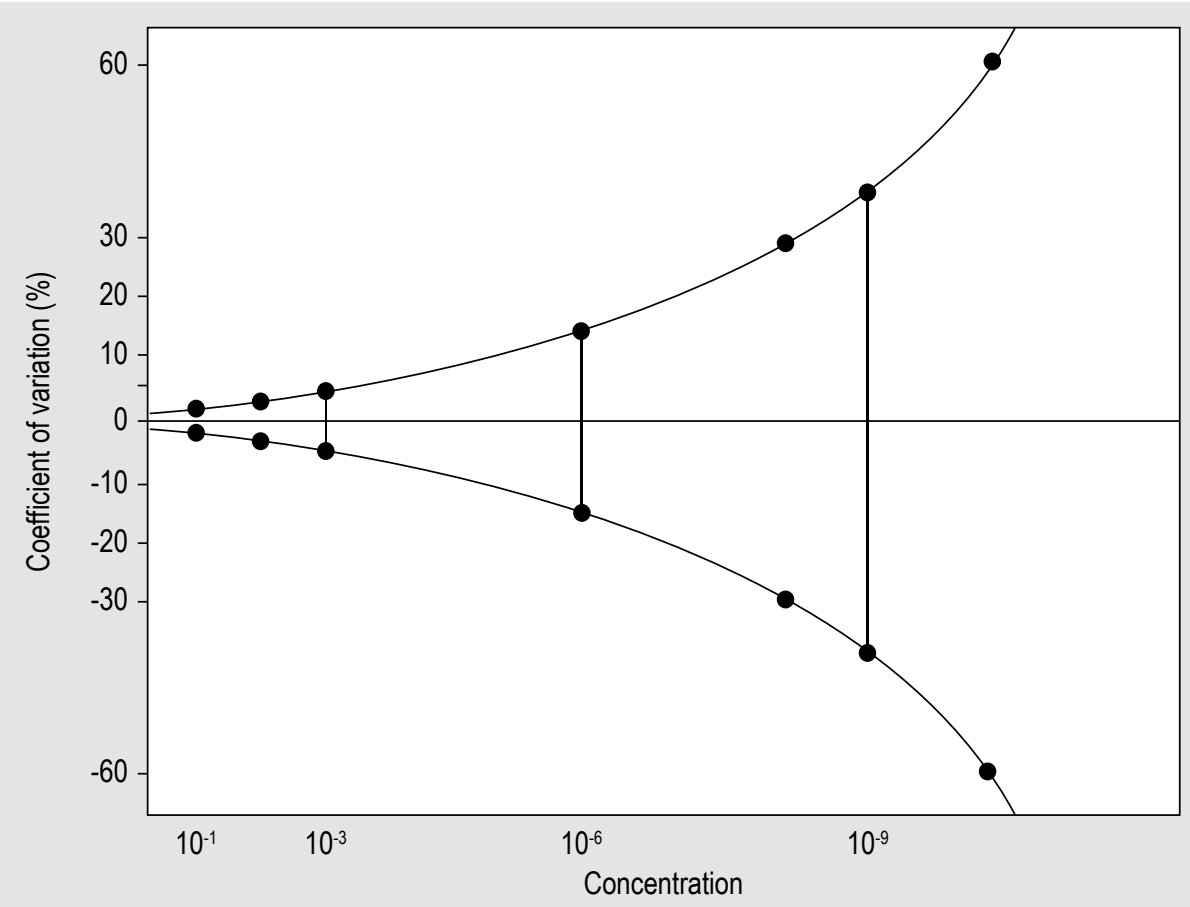

Figure 1. Horwitz Horn, the original curve (after Rivera and Rodriguez, 2013). 


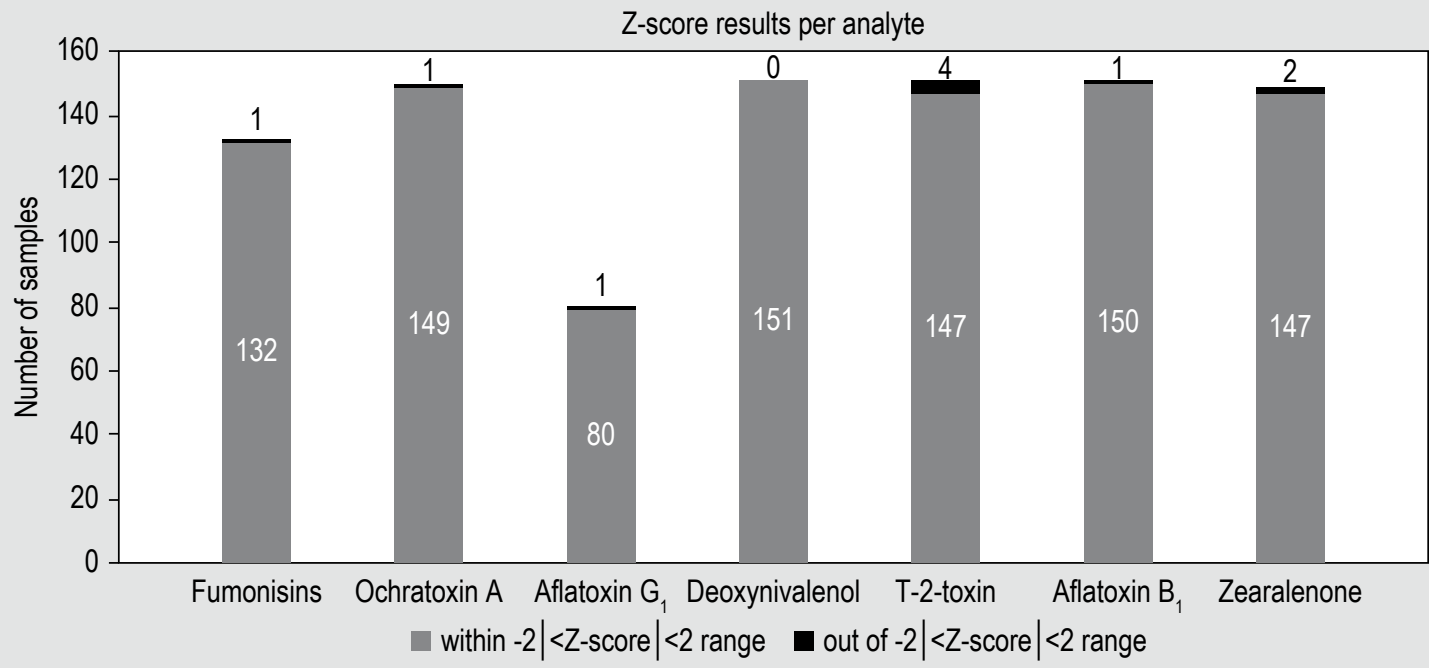

Figure 2. Summary of samples Z-score results per analyte across all 9 laboratories.

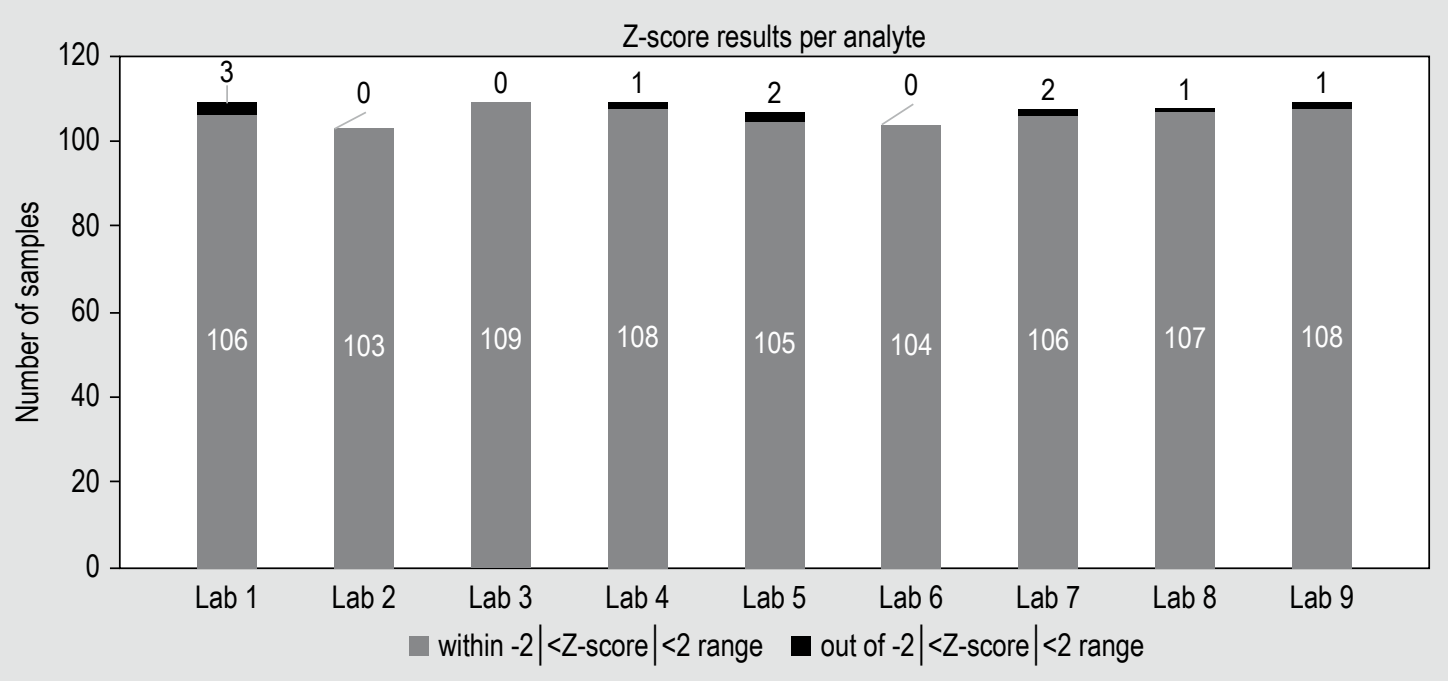

Figure 3. Summary of samples Z-score results per laboratory across all 7 assays.

Reproducibility of Myco 7 kit was also assessed by determination of inter laboratory $\mathrm{CV}$ from mycotoxin concentrations for each sample obtained within a study. Independent analyst in each laboratory repeated the same protocol and after one day of training extracted and tested the same 17 samples. The current reproducibility across AAFCO PT mycotoxin scheme is reaching CVs of $45 \%$ (data from 2020) and the requirement set up at the beginning of study was $<40 \%$. Achieved reproducibility for all 17 samples across 9 laboratories is summarised in Table 3, where the average \% CV differ depending on assay, the lowest \%CV of $16.2 \%$ was obtained for the DON assay and the highest of $19.8 \%$ for $\mathrm{AFB}_{1}$ assay. This shows significant improvement in reproducibility while using Myco 7 biochip array technology.

\section{Discussion}

The routine analysis of mycotoxins within both private and governmental laboratories is challenging due to either the quantity of test kits required like ELISA (enzyme-linked immunosorbent assay) which typically require multiple operators, sample preparations and employ a high number of consumables or the use of liquid chromatography methods, which are expensive to implement, maintain and are typically complex to operate with a heavy training commitment for skilled operators. Due to the limitations of common existing technologies many routine laboratories do not have the capacity to conduct a full mycotoxin analysis on every sample meaning vital information on contamination can be overlooked. Today, the interest and demand in the application of multiplex technology in routine mycotoxin 


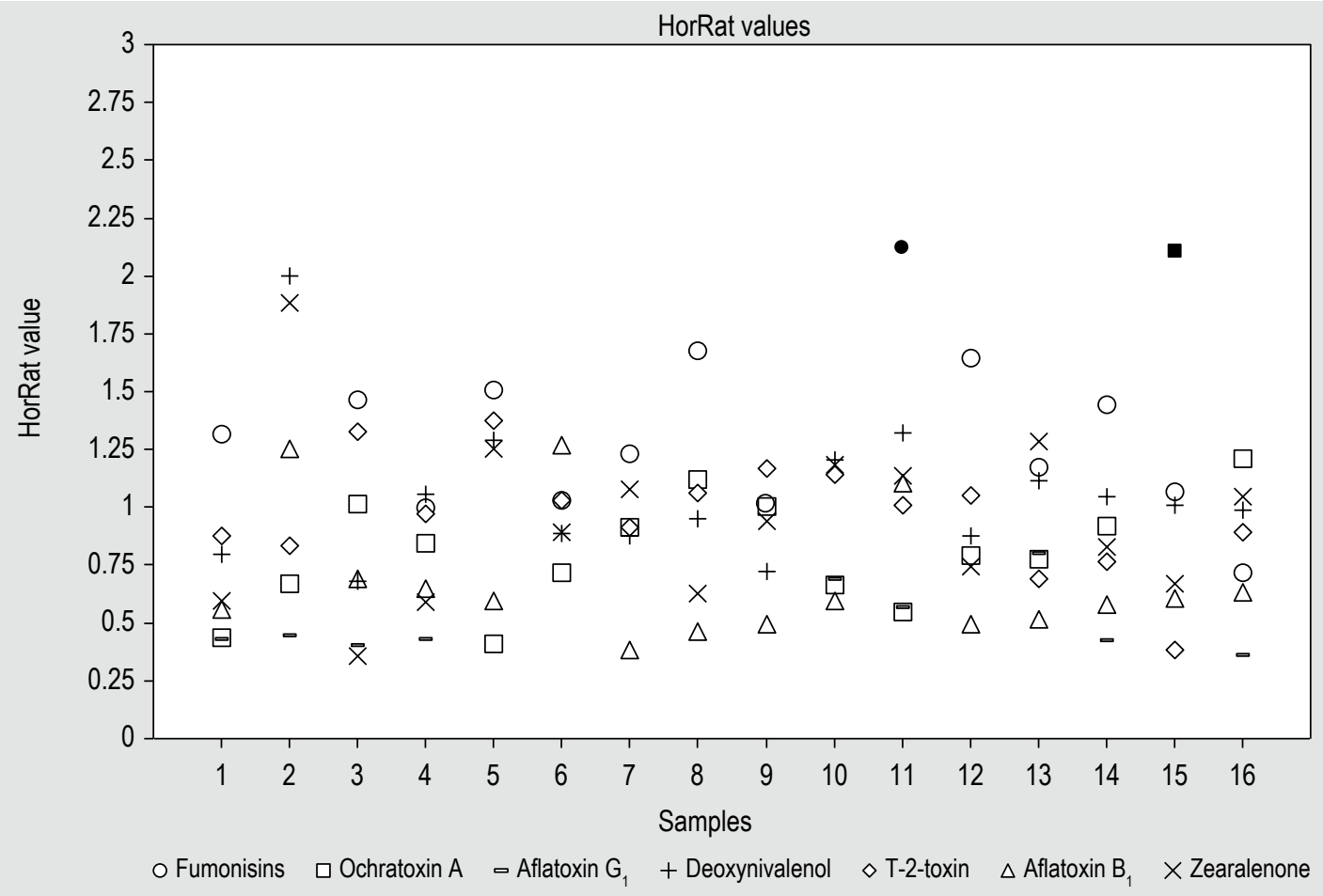

Figure 4. Myco 7 reproducibility presented as HorRat values per sample per analyte. Data points with no inserts or black line as in the legend (101 points) were within set HorRat values criterion $\mathbf{0 . 3 - 2 . 0}$. Data points with black inserts (2 points) were out of set HorRat values criterion 0.3-2.0.

Table 3. Inter laboratory coefficient of variation. ${ }^{1}$

Myco 7 reproducibility - coefficient of variation (\%)

\begin{tabular}{lllllll}
\hline FBs & OTA & AFG $_{1}$ & DON & T-2 & AFB $_{1}$ & ZEN \\
$17.3 \pm 4.2$ & $17.9 \pm 7.9$ & $17.6 \pm 5.7$ & $16.2 \pm 5.0$ & $19.1 \pm 4.6$ & $19.8 \pm 8.9$ & $17.3 \pm 6.4$
\end{tabular}

${ }^{1} \mathrm{AFB}_{1}=$ aflatoxin $\mathrm{B}_{1} ; \mathrm{AFG}_{1}=$ aflatoxin $\mathrm{G}_{1} ; \mathrm{DON}=$ deoxynivalenol; FBs $=$ fumonisins $B_{1}, B_{2}$ and $B_{3} ;$ OTA = ochratoxin $A ; T-2=T-2$ toxin; $Z E N=$ zearalenone.

analysis is growing as indicated by (Munkvold et al., 2019) that detection of multiple toxins within a single assay is desirable.

This set of data is a good illustration of the performance of the Evidence Investigator Myco 7 Array test. The Z-scores demonstrate the high accuracy and reproducibility of the test with overall $99 \%$ pass rate obtained and ranging from 97 to $100 \%$ among 7 mycotoxin assays. This also shows the robustness and ruggedness of the system as analysts had only 1 day of training before running this set of numerous samples. The reproducibility is again illustrated by the HorRat values whose upper range was maintained at a minimum of 0.30 and at s maximum of 2 as recommended by the AOAC (2009). There were 101 from 103 data points, which passed set HorRat value criterion, showing $98 \%$ pass rate, whereas two outstanding points were just out of set range, being 2.11 and 2.13 . However, the high error estimation of the Horwitz formula at low analyte concentrations might have impacted the results for kit control samples as suggested by Linsinger and Josephs (2006) and was taken out of calculations. It is, however, evident from this data that the Evidence Investigator Biochip Myco 7 Array test is fit-for-purpose under feed control testing environments for mycotoxins. Reproducibility described as \%CV across the whole study was between $16-20 \%$ depending on the assay, which confirms that study met the performance requirement of $<40 \%$, as prescribed in AAFCO's Method Needs Statement, for all samples across all 7 predominant mycotoxins groups. Significant improvement to reproducibility results being obtained with current, various methods of analysis within AAFCO PT schemes for the same 7 mycotoxins was also observed.

\section{Conclusions}

The results of this collaborative study support previous multi-mycotoxin validation using the Myco 7 biochip array test kit as described previously (Freitas et al., 2016) and further highlights that a solution to the current challenges in routine mycotoxin analysis exists with the use of Randox Food Diagnostics biochip array technology. Overall, after 
one day of training, all participating AAFCO laboratories found the technology simple to learn and set up. The test procedure of the Myco 7 kit easy to conduct and results generated straight forward to interpret. The importance of accurate multi-mycotoxin analysis is paramount to mitigate negative effects on both animal and human health. This interlaboratory collaborative study demonstrated the reproducibility of the Randox multiplex biochip array for the simultaneous detection of 7 mycotoxins in feed matrices.

\section{Supplementary material}

Supplementary material can be found online at https://doi. org/10.3920/WMJ2021.2696.

Figure S1. Aflatoxin $\mathrm{B}_{1}$ calibration curve.

Figure S2. Aflatoxin $\mathrm{G}_{1}$ calibration curve.

Figure S3. Ochratoxin A calibration curve.

Figure S4. Deoxynivalenol calibration curve.

Figure S5. Zearalenone calibration curve.

Figure S6. Fumonisins calibration curve.

Figure S7. T-2/HT-2 toxin calibration curve.

\section{Conflict of interest}

LS, MP, JP and PF are employed by Randox Food Diagnostics, producer of the Myco 7 biochip.

\section{References}

Alshannaq, A.F. and Yu, J.-H., 2017. Occurrence, toxicity, and analysis of major mycotoxins in food. International Journal of Environmental Research and Public Health 14: 632.

American Association of Feed Control Officials (AAFCO), 2018. Mycotoxins PT Scheme 2020-61. AAFCO, Champaign, IL, USA. Available at: https://tinyurl.com/56an2u3a.

Association of Official Analytical Chemists International (AOAC), 2009. A method for the determination of multiple mycotoxins in feeds and raw grains intended for feeds. Method needs statement and validation criteria. AOAC Agricultura Materials Task Force Sub-Committee on Feed Additives and Contaminants. AOAC, Rockville, MD, USA. Available at: https://www.aafco.org.

Bazin, I., Nabais, E. and Lopez-Ferber, M., 2010. Rapid visual tests: fast and reliable detection of ochratoxin A. Toxins 2: 2230-2241.

Berthiller, F., Cramer, B., Iha, M.H., Krska, R., Lattanzio, V.M.T., MacDonald, S., Malone, R.J., Maragos, C., Solfrizzo, M., StranskaZacharisova, M., Stroka, J. and Tittlemier, S.A., 2018. Development in mycotoxin analysis: an update for 2016-2017. World Mycotoxin Journal 11: 5-31.
Biomin, 2019. World mycotoxin survey 2019. Available at: https:// www.biomin.net/solutions/mycotoxin-survey.

Curticapean, A., Toma, F., Tarcea, M., Curticapean, M., Samarghitan, V., Pop, A.I. and Gulea, A., 2011. HPLC method validation for simultaneous determination of three mycotoxins from corn seeds. Croatia Chemica Acta 84: 413-417.

De Girolamo, A., Ciasca, B., Stroka, J., Bratinova, S., Pascale, M., Visconti, A. and Lattanzio, V.M.T., 2017. Performance evaluation of LC-MS/MS methods for multi-mycotoxin determination in maize and wheat by means of international proficiency testing. Trends in Analytical Chemistry 86: 222-234.

European Commission (EC), 2002. 2002/657/EC: Commission Decision of 12 August 2002 implementing Council Directive 96/23/ EC concerning the performance of analytical methods and the interpretation of results. Official journal of the European Union L 221: 8-36.

FitzGerald, S.P., Lamont, J.V., McConnell, R.I. and Benchihk, E.O., 2005. Development of a high-throughput automated analyser using biochip array technology. Clinical Chemistry 51: 1165-1176.

Freitas, A., Brites, C., Barbosa, J., Sanches-Silva, A. and Barros, S., 2016. Validation of a biochip chemiluminescent immunoassay for multi-mycotoxins screening in maize (Zea mays L.). Food Analytical Methods 12: 2675-2684.

Gaudin, V., Hedou, C., Soumet, C. and Verdon, E., 2014. Evaluation and validation of Biochip multi-array technology for the screening of six families of antibiotics in honey according to the European guideline for the validation of screening methods for residues of veterinary medicines. Food Additives and Contaminants Part A 31: 1699-1711.

Horwitz, W., 1994. Protocol for the design, conduct and interpretation of method-performance studies: Technical Report. Interdivisional Working Party on Harmonization of Quality Assurance Schemes for Analytical Laboratories. International Union of Applied and Pure Chemistry. Pure and Applied Chemistry 67: 331-343.

Irakli, M.N., Skendi, A. and Papageorgiou, M.D., 2017. HPLC-DADFLD method for simultaneous determination of mycotoxins in wheat bran. Journal of Chromatographic Science 55: 690-696.

International Organization for Standardization (ISO), 1994. ISO 5725:1994. Precision of test methods - Part 2: basic method for the determination of repeatability and reproducibility. Available at: https://www.iso.org/standard/11833.html

Linsinger, T.P.J. and Josephs, R.D., 2006. Limitations of the application of the Horwitz equation. Trends in Analytical Chemistry 25: 11251130.

Krska, R. and Molinelli, A., 2008. Rapid test strips for analysis of mycotoxins in food and feed. Analytical and Bioanalytical Chemistry 393: 67-71.

Munkvold, G., Arias, S., Taschl, I. and Gruber-Dorninger, C., 2019. Mycotoxins in corn: occurrence, impacts, and management. In: Serna-Saldivar, S.O. (ed.) Corn - chemistry and technology. AACC International Press, Washington, DC, USA, pp. 235-287.

Porter, J., O’Loan, N., Bell, B., Mahoney, J., McGarrity, M., McConnell, R.I. and Fitzgerald, S.P. 2012. Development of an evidence biochip array kit for the multiplex screening of more than 20 anthelmintic drugs. Analytical and Bioanalytical Chemistry 403: 3051-3056. 
Plotan M., Devlin R., Porter, J., El Ouard Benchikh, M., Rodríguez M., McConnell, I. and Fitzgerald, P., 2016. The use of biochip array technology for rapid multi-mycotoxin screening. Journal of AOAC International 99: 878-888.

Rivera, C. and Rodríguez, R., 2013. Horwitz equation as quality benchmark in ISO/IEC 17025 testing laboratory. Available at: https://www.bii.mx/documentos/horwitzCf11.pdf.

Rodrigues, I. and Naehrer, K., 2012. A three-year survey on the worldwide occurrence of mycotoxins in feedstuffs and feed. Toxins 4: 663-675.
Streit, E., Schatzmayr, G., Tassis, P., Tzika, E., Marin, D., Taranu, I., Tabuc, C., Nicolau, A., Aprodu, I., Puel, O. and Oswald, I.P., 2012. current situation of mycotoxin contamination and co-occurrence in animal feed - focus on Europe. Toxins 4: 788-809.

Taverniers, I., De Loose, M. and Van Bockstaele, E., 2004. Trends in quality in the analytical laboratory. II. Analytical method validation and quality assurance. Trends in Analytical Chemistry 23: 535-552.

Tittlemier, S., Cramer, B., Dall'Asta, C., Iha, M., Lattanzio, V., Malone, R., Maragos, C., Solfrizzo, M., Stranska-Zachariasova, M. and Stroka, J., 2019. Developments in mycotoxin analysis: an update for 2017-2018. World Mycotoxin Journal 12: 3-29. 